\title{
THE RHODES/HARTRAO 2300 MHZ HORN TELESCOPE
}

\author{
J.L. JONAS \\ Rhodes University, Grahamstown 6140, South Africa \\ phjj@hippo.ru.ac.za
}

\begin{abstract}
The recently completed Rhodes/HartRAO $2300 \mathrm{MHz}$ radio continuum survey is the highest resolution and highest frequency survey of the entire southern sky. This makes it useful for those wishing to model the Galactic foreground in order to study cosmic background emission. Unfortunately there are low-amplitude, large-scale gradients in the survey data which make it difficult to interpret the very high latitude Galactic emission. We describe a small, low-cost horn telescope which we hope to use to correct the gradients in the survey data.
\end{abstract}

\section{Introduction}

The estimation of the Galactic foreground contribution to measurements of the CMB is becoming very important now that positive detections of multipole anisotropies are being reported. Large-scale centimetre-wavelength radio continuum surveys at a number of frequencies are necessary to obtain accurate information about the spatial distribution of the brightness and spectral index of the Galactic emission. Results from these surveys may be extrapolated to the millimetre-wavelength regime of CMB measurements.

The Rhodes/HartRAO $2300 \mathrm{MHz}(13 \mathrm{~cm})$ survey (Jonas, Baart \& Nicolson, in preparation) provides unique centimetre-wavelength coverage of the southern hemisphere. Being the highest-frequency (and resolution) radio continuum survey made of the southern sky using a terrestrial telescope it is well suited to the extrapolation necessary to estimate Galactic emission at higher frequencies. The large range of extrapolation places stringent requirements on the calibration and baseline accuracy of the low frequency data. Large-scale gradients in the $2300 \mathrm{MHz}$ data introduce relative baseline errors of up to $50 \mathrm{mK}$, and the brightness temperature calibration is of

M. Kafatos and Y. Kondo (eds.), Examining the Big Bang and Diffuse Background Radiations, 487-488. (C) 1996 IAU. Printed in the Netherlands. 
order $5 \%$. In order to improve these parameters of the survey it was decided that unblocked aperture measurements had to be made.

\section{Telescope Structure}

The telescope is constructed almost entirely from wood to ensure low cost. The horn antenna and all of the electronic instrumentation are contained within a rectangular "tube", which is internally clad with polystyrene sheets for thermal insulation. The tube may be fixed at any elevation angle, and the alidade has a motorized azimuth drive which allows computer control. The telescope baseplate is mounted on a road trailer, allowing the telescope to be transported between different sites.

\section{Instrumentation}

Currently the telescope uses a simple rectangular horn antenna, which has a HPBW of $15^{\circ}$ in both the E- and H-planes. The sidelobe performance is not well suited to this experiment, but funds are not available for a purpose-built horn. Ambient temperature GaAsFET amplifiers provide about $60 \mathrm{~dB}$ front-end gain to the receiver. The radiometer is a noise adding, gain-stabilized design which is well suited to provide the stability necessary for this experiment. All of the receiver components are housed in the "tube", where the air temperature is controlled to within $0.1^{\circ} \mathrm{C}$.

A PC-type computer controls all aspects of the telescope operation, allowing unattended all-night observations. Modular design of the instrumentation ensures that the telescope can be set up quickly at the observing site.

\section{Technique}

The experiment has been designed to make differential sky temperature measurements, using the south celestial pole (SCP) as a reference point. The antenna is fixed at the elevation of the SCP and made to scan alternately clockwise and anti-clockwise by $360^{\circ}$ in azimuth. Each scan consists of slewing steps between positions on a $5^{\circ}$ declination grid, and each scan begins and ends at the SCP reference position. The sky temperature is integrated for 10 seconds at each position, resulting in one complete $360^{\circ}$ scan taking about 12 minutes. The short scan time allows baseline drifts to be removed by linear interpolation of the SCP temperatures.

By stacking the data from observations made throughout the year and from different sites we will obtain a low resolution map of the sky brightness relative to the SCP. The main survey data will be convolved with an appropriate beam so that it may be compared with the horn data. 